\title{
Quantification of organic volatile impurities in Oseltamivir phosphate drug substances by head space gas chromatography
}

\author{
Chandrasekhar Reddy Gudibanda ${ }^{1,4^{*}}$ (D), Shyamala Pulipaka², Murali Krishna Rallabhandi ${ }^{3}$, \\ Maruthi Venkata Narayana Rao Kapavarapu and Mannem Durga Babu Mannem
}

\begin{abstract}
Background: The present work is aimed at quantification of organic volatile solvents like Methanol, Ethanol, Acetonitrile, Isopropyl alcohol (IPA), Dichloromethane (DCM), Methyl tert-butyl ether (MTBE), Hexane fractions, Ethyl acetate (EA), Tetra hydro furan (THF), 1,4-Dioxane, Dimethylformamide (DMF), and Toluene in Oseltamivir phosphate Active pharmaceutical ingredient (API) and pharmaceutical dosage forms. The method was developed using a thermal gradient elution program associated with a column having dimensions are DB-1,60 $\mathrm{m} \times 0.32 \mathrm{~mm} \times 5.0 \mu \mathrm{m}$ with a flow rate of $1.0 \mathrm{~mL} / \mathrm{min}$ and Nitrogen $\left(\mathrm{N}_{2}\right)$ as a carrier gas. A flame ionization detector was used as a detector, and its temperature is at $290^{\circ} \mathrm{C}$ whereas the injector temperature is at $180^{\circ} \mathrm{C}$. The total run time is $60.0 \mathrm{~min}$. The developed method was validated according to International Council for Harmonization $(\mathrm{ICH})$ guidelines.

Results: The linearity of the calibration curve for twelve impurities in the concentration range of Limit of Quantification (LOQ) to 150\% was good. The curve was linear for twelve residual impurities of Oseltamivir phosphate. Relative standard deviation values for twelve residual impurities are not more than 15\%. Limit of detection (LOD) and LOQ for twelve residual impurities were found to be very low-level concerning specification level. We found good results for all validated parameters for twelve residual impurities.

Conclusions: To quantify the residual organic volatile solvents (organic volatile impurities) in the Oseltamivir phosphate API, a novel, specific, and elevated sensitive headspace gas chromatography method was developed to separate twelve solvents that are accompanying with fifteen peaks. Out of fifteen peaks, critical components are Hexane fractions, Ethyl acetate, MTBE, and DCM. So, our method has capable of separating and simultaneous quantification such critical components. So, it can be successfully applied for routine analysis of quantification of these twelve residual impurities in Oseltamivir phosphate bulk and pharmaceutical dosage forms.
\end{abstract}

Keywords: Oseltamivir phosphate, Organic volatile impurities, Method development, Method validation

\section{Background}

Residual solvents in pharmaceuticals are defined here as organic volatile chemicals that are used or produced in the manufacture of drug substances or excipients, or the preparation of drug products. The solvents are not

\footnotetext{
${ }^{*}$ Correspondence:

1 GVK Biosciences Pvt. Ltd, Hyderabad, Telangana, India

Full list of author information is available at the end of the article
}

completely removed by practical manufacturing techniques. Appropriate selection of the solvent for the synthesis of drug substance may enhance the yield, or determine characteristics such as crystal form, purity, and solubility. Therefore, the solvent may sometimes be a critical parameter in the synthetic process. Since there is no therapeutic benefit from residual solvents, all residual solvents should be removed to the extent possible to meet product specifications, good manufacturing practices, or other quality-based requirements. Drug products should 
contain no higher levels of residual solvents than can be supported by safety data. The organic volatile impurity specifications are set by the ICH guidelines, regulatory authorities, and those guidelines are established based on respective toxicology data of solvents, and it is varying from a low-level part per million ( $\mathrm{ppm}$ ) to thousands of ppm based on the classification of residual solvents by risk assessment. To quantify the organic volatile impurities gas chromatographic instrument connected with the headspace instrument is being used more effectively, and it is playing a vital role in the pharmaceutical industry [1-6]. To enhance the quality as well as quantity of Oseltamivir Phosphate API, the following solvents were used as part of the synthetic process. To make affordable Oseltamivir Phosphate API, Hexane fractions are being used instead of $\mathrm{n}$-Hexane solvent. Which is accomplished with mainly four fractions, and which is a critical part of development since these fractions are being co-eluted with MTBE as well as Ethyl acetate solvents, hence the efforts were made to separate the critical components like Hexane fractions, MTBE, Dichloromethane, Ethyl acetate along with other solvents. Many papers are published to quantify the residual solvents in the drug substance, but no method is readily available to separate such critical solvents.

Oseltamivir phosphate is a white crystalline solid with the chemical name (3R,4R,5S)-4-acetylamino5-amino-3(1-ethylpropoxy)-1-cyclohexene-1-carboxylic acid ethyl ester, phosphate. The chemical formula is $\mathrm{C}_{16} \mathrm{H}_{28} \mathrm{~N}_{2} \mathrm{O}_{4}$ (free base), and the molecular weight of Oseltamivir is $312.4 \mathrm{gr} / \mathrm{mol}$. The chemical formula is $\mathrm{C}_{16} \mathrm{H}_{31} \mathrm{~N}_{2} \mathrm{O}_{8} \mathrm{P}$ (salt), and the molecular weight of Oseltamivir is $410.4 \mathrm{gr} / \mathrm{mol}$ for Oseltamivir phosphate salt. The structural formula is shown in Fig. 1.

Different organic solvents are used in the synthetic process of Oseltamivir phosphate API. The organic solvents like Methanol, Ethanol, Acetonitrile, Isopropyl alcohol, Dichloromethane, MTBE, Hexanes, Ethyl acetate, THF, 1,4-Dioxane, DMF, and Toluene. As per ICH Q3C (R6) Ethanol, Isopropyl alcohol, MTBE, Ethyl acetate, and DMF were grouped under class- 3 organic solvents while Methanol, Acetonitrile, Dichloromethane, Hexanes,

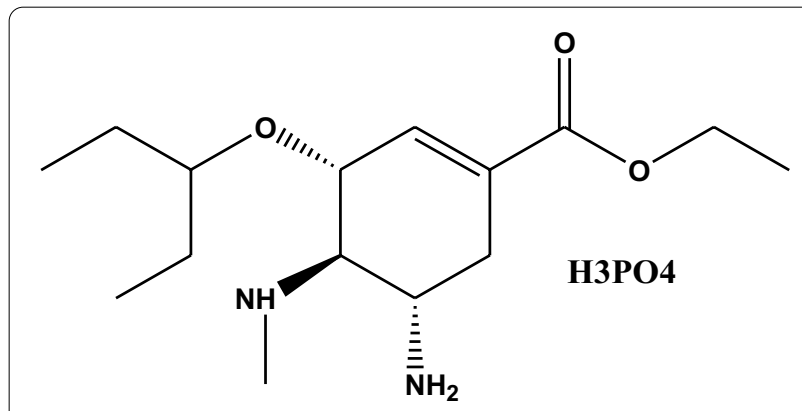

Fig. 1 Chemical structure of Oseltamivir phosphate API
THF and 1,4-Dioxane, and Toluene were grouped under class-2 organic solvents [7].

Organic volatile impurities (OVI) or solvents of class-2 have inherent toxicity to human wellbeing, and chemical solvents of class- 3 are less harmful. Therefore, the organic solvents utilized in Oseltamivir phosphate API must be regulated. The ICH Q3 specification levels opt for twelve organic volatile impurities in Oseltamivir phosphate API. Developed a novel and highly sensitive method to quantify the organic volatile impurities such as Methanol, Ethanol, Acetonitrile, Isopropyl alcohol, Dichloromethane, MTBE, Hexanes, Ethyl acetate, THF, 1,4-Dioxane, DMF, and Toluene. This is a novel method since the key component is to separate the critical solvents such as MTBE, Dichloromethane, Ethyl acetate, and Hexanes (Fraction-1,2,3 and 4) are in a single analysis. So, our proposed method should be separate these combinations along with other solvents, and we got good resolution between twelve organic volatile impurities accomplished with fifteen peaks and then validated as per ICH grainlines and Association of Official Agricultural Chemists (AOAC) guidelines. This method is very novel, simple, accurate, and precise.

\section{Literature survey}

Few chromatographic methods have appeared in the literature. Those are the development and validation of the Reversed-phase High-performance liquid chromatography (RP-HPLC) method for the determination of Oseltamivir phosphate in bulk drug and dosage forms [8]. Stability indicates the liquid chromatography (LC) method for Oseltamivir phosphate [9]. The degradation behavior of Oseltamivir phosphate under various stress conditions was used stability-indicating HPLC method [10]. A literature search revealed that nobody has reported the quantification of Organic volatile impurities also. We have well separated Dichloromethane, Ethyl acetate MTBE with Hexanes fractions combination. So, our proposed gas chromatography-headspace (GC-HS) method is novel and very sensitive.

\section{Methods}

\section{Chemicals and reagents}

Oseltamivir phosphate API was taken from GVK Biosciences Pvt. Ltd. (Hyderabad, India).

The solvents viz. methanol, ethanol, acetonitrile, isopropyl alcohol, Dichloromethane, MTBE, Hexanes, ethyl acetate, THF, 1,4-Dioxane, DMF, and Toluene (all procured from Merck, India).

\section{Instruments}

Agilent GC 6890 N system, Agilent headspace G1888 N system, Flame ionization detector system, Waters United 
States of America (USA) Empower version 3 software were used for the quantification of opted Twelve organic volatile solvents in Oseltamivir phosphate API.

\section{Chromatographic conditions}

\begin{tabular}{lll}
\hline Column & $:$ & $\mathrm{DB}-1,60 \mathrm{~m}, 0.32 \mathrm{~mm}$, and $5.0 \mathrm{\mu m}$ \\
Injector temperature & $:$ & $180^{\circ} \mathrm{Centigrade}\left({ }^{\circ} \mathrm{C}\right)$ \\
Detector & $:$ & Flame ionization detector (FID) \\
Detector temperature & $:$ & $290^{\circ} \mathrm{C}$ \\
Initial oven temperature & $:$ & $40^{\circ} \mathrm{C}$ \\
Hold time-1 & $:$ & $10.0 \mathrm{~min}$ \\
Ramp rate-1 & $:$ & $5.0^{\circ} \mathrm{C} / \mathrm{min}$ \\
Oven temperature & $:$ & $80^{\circ} \mathrm{C}$ \\
Hold time-2 & $:$ & $10.0 \mathrm{~min}$ \\
Ramp rate-2 & $:$ & $4.0^{\circ} \mathrm{C} / \mathrm{min}$ \\
Oven temperature & $:$ & $150^{\circ} \mathrm{C}$ \\
Hold time-3 & $:$ & $5.0 \mathrm{~min}$ \\
Ramp rate-3 & $:$ & $40.0^{\circ} \mathrm{C} / \mathrm{min}$ \\
Oven temperature & $:$ & $280^{\circ} \mathrm{C}$ \\
Hold time-4 & $:$ & $6.25 \mathrm{~min}$ \\
Carrier gas & $:$ & Nitrogen \\
Mode of injection & $:$ & $\mathrm{Split}$ \\
Constant Flow & $:$ & $1.0 \mathrm{~mL} / \mathrm{min}$ \\
Split ratio & $:$ & $1: 5$ \\
Makeup & $:$ & $30 \mathrm{~mL} / \mathrm{min}$ \\
Hydrogen & $:$ & $40 \mathrm{~mL} / \mathrm{min}$ \\
Air & $:$ & $400 \mathrm{~mL} / \mathrm{min}$ \\
Run time & $:$ & $60.0 \mathrm{~min}$ \\
Diluent & $\mathrm{N}-\mathrm{methyl}-2-$-pyrrolidone (NMP) \\
\hline
\end{tabular}

Headspace parameters

\begin{tabular}{lll}
\hline Oven temperature & $:$ & $90^{\circ} \mathrm{C}$ \\
Loop temperature & $:$ & $160^{\circ} \mathrm{C}$ \\
Transfer line temperature & $:$ & $165^{\circ} \mathrm{C}$ \\
GC cycle time & $:$ & $75 \mathrm{~min}$ \\
Injection time & $:$ & $5.0 \mathrm{~min}$ \\
Loop equilibration time & $:$ & $0.05 \mathrm{~min}$ \\
Loop fill time & $:$ & $2.0 \mathrm{~min}$ \\
Pressurization & $:$ & $2.0 \mathrm{~min}$ \\
Vial equilibration & $:$ & $30.0 \mathrm{~min}$ \\
Shaking & $:$ & High \\
Vial pressure & $:$ & $\sim 15$ Pounds per \\
& & square inch (psi) \\
& & (Nitrogen) \\
\hline
\end{tabular}

Specifications of organic twelve volatile impurities
Methanol

Not more than 3000 ppm

Ethanol

Isopropyl alcohol

Dichloromethane

Hexanes

Ethyl acetate

THF

Toluene

DMF

1.4-Dioxane

Acetonitrile

MTBE

Not more than 5000 ppm

Not more than 5000 ppm

Not more than 600 ppm

Not more than 290 ppm

Not more than 5000 ppm

Not more than 720 ppm

Not more than 890 ppm

Not more than 880 ppm

Not more than 380 ppm

Not more than $410 \mathrm{ppm}$

Not more than 5000 ppm

\section{Standard and sample solution preparations}

Blank preparation Transferred 2.0 Milley Letter (mL) of diluent into a $20 \mathrm{ml}$ headspace vial. Immediately crimped the vial with a septum and a cap.

\section{Standard stock preparation}

Weighed and transferred about $600 \mathrm{mg}$ of Methanol, $1000 \mathrm{mg}$ of Ethanol, $82 \mathrm{mg}$ of Acetonitrile, $1000 \mathrm{mg}$ of IPA, $120 \mathrm{mg}$ of DCM, $1000 \mathrm{mg}$ of MTBE, $58 \mathrm{mg}$ of Hexanes, $1000 \mathrm{mg}$ of Ethyl acetate, $144 \mathrm{mg}$ of THF, $76 \mathrm{mg}$ of 1,4-Dioxane, $176 \mathrm{mg}$ of DMF, and $178 \mathrm{mg}$ of Toluene into a $50 \mathrm{~mL}$ volumetric flask contained $20 \mathrm{~mL}$ of diluent dissolved and diluted to volume with diluent.

Standard preparation Transferred $1.0 \mathrm{~mL}$ of the stock solution into $100 \mathrm{~mL}$ volumetric flask containing $50 \mathrm{~mL}$ of diluent, dissolved, and diluted to volume with diluent.

Preparation of standard vial Transfer $2.0 \mathrm{~mL}$ of standard stock solution in a headspace vial and seal with an aluminum septum and crimp the cap.

\section{Preparation Oseltamivir phosphate sample}

Accurately weighed and transferred 80 Milly gram (mg) of Oseltamivir phosphate into a headspace vial. Then add $2.0 \mathrm{~mL}$ of diluent and immediately seal with an aluminum septum and crimp the cap.

\section{Pharmaceutical sample preparation}

Twenty tablets were weighed and powdered. Accurately weighed and transferred an amount of powder equivalent to $80 \mathrm{mg}$ of Oseltamivir phosphate to a $20 \mathrm{~mL}$ headspace vial then added $2.0 \mathrm{~mL}$ of diluent and immediately seal with an aluminum septum and crimp the cap.

The Organic volatile impurity content (ppm) was calculated by the following formula: 


$$
\text { Calculation (ppm): } \frac{\text { Impurity area in test solution }}{\text { Impurity area in standard solution }} \times \frac{\text { Standard concentration }(\mathrm{mg} / \mathrm{mL})}{\text { Sample concentration }(\mathrm{mg} / \mathrm{mL})} \times 10^{6}
$$

\section{GC-HS method development}

The adopted solvents for the Oseltamivir phosphate API are associated with class-II and class-III solvents by ICH guidelines. To develop an elevated sensitive and novel method, one needs to choose the best suitable solvent and column for Oseltamivir phosphate. The method should be robust to determine the trace levels of residual solvents in the drug substance as well as the drug product. As part of method development, following the quality by design principles to have a better control method for residual solvents in Oseltamivir phosphate.

\section{Diluent selection}

This method development was started with the selection of diluent. We have sought different diluents (DMF, NMP, and Dimethyl sulfoxide). For its ability to dissolve a broad range of organic solvents and will not impede with chosen solvents, analyzed by gas chromatography, NMP was exploited as the standard as well as sample diluent.

\section{Column selection}

The Column selection for GC-HS analysis was also a very important task in the method development process. This study utilized a chromatographic basic rule "like attracts like" and focused on the polarity matching among column stationary phase and mobile phase. In this study, many columns were screened i.e., VF-1 $(30 \mathrm{~m} \times 0.32 \mathrm{~mm} \times 0.45 \mu \mathrm{m}), \quad$ DB-624 $(30 \mathrm{~m} \times 0$. $53 \mathrm{~mm} \times 3.0 \mu \mathrm{m}), \mathrm{DB}-1,60 \mathrm{~m}, 0.32 \mathrm{~mm} \times 5.0 \mu \mathrm{m}$ and DB-624 $(30 \mathrm{~m} \times 0.25 \mu \mathrm{m} \times 0.25 \mu \mathrm{m})$, DB-17, DB-5, DB-Vax columns. Out of seven columns , DB-1 column gave better resolution between Ethyl acetate, MTBE, Dichloromethane, and Hexanes whereas in other columns VF-1, DB-624, DB-5, DB-Vax either MTBE peak is co-eluting with Hexanes first peak or with DCM peak. In some columns, Ethyl acetate is co-eluting with Hexane's fraction 3. The GC-HS parameters were first optimized to achieve good retention time, acceptable resolution, and better peak shapes for the MTBE, Hexanes, Dichloromethane, and Ethyl acetate in Oseltamivir phosphate and its formulations. The DB-1 eluted sharp peaks with good peak tailing and good resolution between all peaks. It demonstrated that the DB-1 column was closely matched. Hence, the DB-1 $60 \mathrm{~m}$, $0.32 \mathrm{~mm} \times 5.0 \mu \mathrm{m}$ column was selected for this study.

\section{Column oven temperature optimization:}

Four different column oven temperature fluxes were tried. (1) maintained for $11 \mathrm{~min}$ at $40{ }^{\circ} \mathrm{C}$ and then continued to upsurge to a temperature close of $240{ }^{\circ} \mathrm{C}$ at a rate of $20^{\circ} \mathrm{C} / \mathrm{min}$ and retained for $30 \mathrm{~min}$; (2) maintained for $10 \mathrm{~min}$ at $40{ }^{\circ} \mathrm{C}$ and then raise $80^{\circ} \mathrm{C}$ with Ramp rate $5.0{ }^{\circ} \mathrm{C} / \mathrm{min}$ hold for $10 \mathrm{~min}$ and again raise $150{ }^{\circ} \mathrm{C}$ with Ramp rate $4.0^{\circ} \mathrm{C} / \mathrm{min}$ hold for $5 \mathrm{~min}$ continued to upsurge to a temperature close of $280^{\circ} \mathrm{C}$ at a rate of $40{ }^{\circ} \mathrm{C} / \mathrm{min}$ and retained for $6.25 \mathrm{~min}$. (3) maintained for $5 \mathrm{~min}$ at $40^{\circ} \mathrm{C}$ and then continued to upsurge to a temperature close of $240{ }^{\circ} \mathrm{C}$ at a rate of $10^{\circ} \mathrm{C} / \mathrm{min}$ and retained for $35 \mathrm{~min}$; (4) maintained for $11 \mathrm{~min}$ at $40{ }^{\circ} \mathrm{C}$ and then continued to upsurge to a temperature close of $240{ }^{\circ} \mathrm{C}$ at a rate of $15{ }^{\circ} \mathrm{C} / \mathrm{min}$ and retained for $30 \mathrm{~min}$;

Better separation with the good resolution was obtained with $1^{\text {st }}$ oven program [maintained for $10 \mathrm{~min}$ at $40{ }^{\circ} \mathrm{C}$ and then raise $80^{\circ} \mathrm{C}$ with Ramp rate $5.0^{\circ} \mathrm{C} / \mathrm{min}$ hold for $10 \mathrm{~min}$ and again raise $150{ }^{\circ} \mathrm{C}$ with Ramp rate of $4.0^{\circ} \mathrm{C} / \mathrm{min}$ hold for $5 \mathrm{~min}$ continued to upsurge to a temperature close of $280^{\circ} \mathrm{C}$ at a rate of $40^{\circ} \mathrm{C} / \mathrm{min}$ and retained for $6.25 \mathrm{~min}$ ]. In $1^{\text {st }}, 3^{\text {rd }}$, and 4 th oven temperature fluxes, the peaks of all the nine organic solvents were closely eluted. Nitrogen, as a carrier gas, with a flowing stream of $1.0 \mathrm{~mL}$ per min and $2 \mathrm{~mL}$ per min were tested. A $1.0 \mathrm{~mL}$ per min flow stream was optimized. The remaining optimized parameters were 180 ${ }^{\circ} \mathrm{C}$ temperature at injector port; $290{ }^{\circ} \mathrm{C}$ temperature at detector port; split mode injection in 1:5 ratio; air flow and hydrogen flow were $400 \mathrm{~mL} / \mathrm{min}$ and $40 \mathrm{~mL} /$ min, respectively. Figure 2 displays the chromatogram acquired using configured parameters.

\section{Results \\ Method validation}

The GC-HS method was validated as per ICH guidelines [11]. The following validation parameters are done. Those are specificity, repeatability, method precision, LOD and LOQ, linearity, accuracy, ruggedness, and robustness.

\section{Specificity and selectivity}

Injected separately the individual standard solutions of each solvent, sample solution, and then selectivity solution. No peak from blank at Methanol, Ethanol, Acetonitrile, Isopropyl alcohol, DCM, MTBE, Hexanes, 


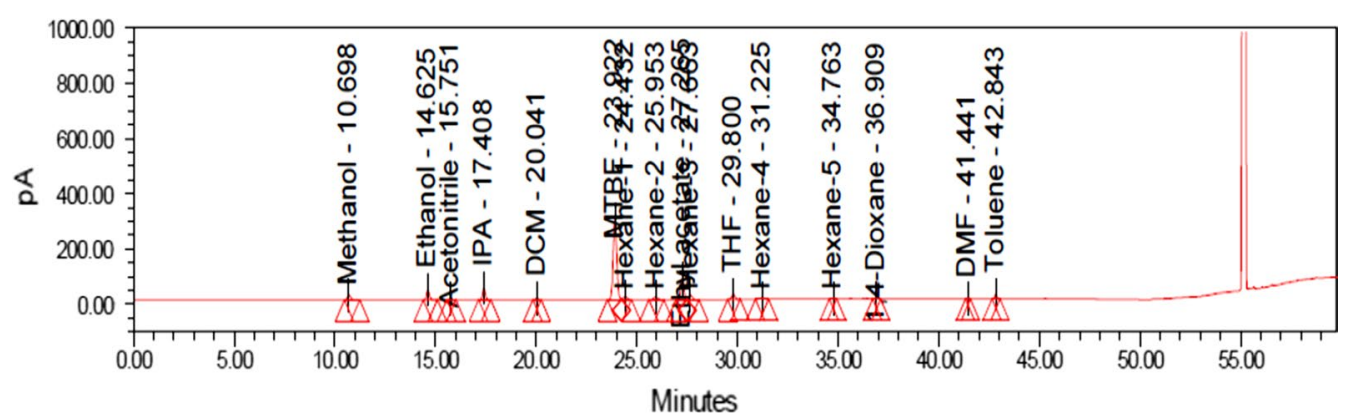

Fig. 2 Typical chromatogram for Twelve organic volatile impurities

Ethyl acetate, THF, 1,4-Dioxane, DMF, and Toluene Retention times. Methanol, Ethanol, Acetonitrile, Isopropyl alcohol, DCM, MTBE, Hexanes, Ethyl acetate, THF, 1,4-Dioxane, DMF, and Toluene solvents should be eluted at different retention times. Reported the resolution between all solvent peaks in specificity solution and reported the retention times of all solvents in individual solutions and selectivity solution. The resolution was obtained between the all peaks was not less than 1.0 , and the typical chromatograms and retention times are shown in Fig. 3a-d and Table 1.

\section{System precision}

The system precision of this method was expressed in terms of the \% relative standard deviation (RSD) of the data. System precision has been demonstrated by six replicate injections of standard solutions. The \% RSD was found out to be not more than $15 \%$. The data of system precision are shown in Table 2.

\section{Method precision}

The method precision of the proposed method is expressed in the term of \% RSD of the data. Method precision has been demonstrated by separately analyzing sample six preparations as per the method. The \%RSD was found to be less than $15 \%$. The data of method precision are shown in Table 3.

\section{LOD and LOQ}

Limit of Detection and Quantitation was established by the signal-to-noise ratio. During the LOD determination, the $\mathrm{s} / \mathrm{n}$ ratio of methanol and acetonitrile was observed as 10.5 and 27.1, in LOQ determination as 10.1 and 33.1, respectively, while the remaining solvents are meeting the acceptance criteria. United states pharmacopeia (USP) signal to noise ( $\mathrm{s} / \mathrm{n}$ ) ratio for LOD should be Greater than or equal $(\geq)$ to 3 and for LOQ should be $\geq 10$ for each specified solvent. The data of LOD and LOQ are presented in Table 4.

\section{Linearity}

The linearity solutions were prepared for each organic volatile impurity over the range of $\mathrm{LOQ}, 50,80,100,120$, and $150 \%$. To draw the linearity graph between concentration and area of organic volatile impurities. Finally, the correlation coefficient $(r)$ was obtained not less than 0.99 for twelve organic volatile impurities. The correlation is calculated based on $Y=M X+c$. The method was linear from LOQ\% level to $150 \%$ level concerning the Oseltamivir phosphate API concentration. The linearity data and typical graph are presented in Table 5 and Fig. $4 \mathrm{a}, \mathrm{b}$.

\section{Accuracy}

Accuracy is performed by the known amount of organic volatile impurities that standard solution was spiked to Oseltamivir phosphate API at three different concentrations $(50,100,150$, and LOQ\%). From these accuracy data, the \% recovery of organic volatile impurities was obtained $100 \pm 20 \%$ at $50 \%$ Level to $150 \%$ Level and $100 \pm 30 \%$ at LOQ Level. The results are shown in Table 6.

\section{System precision at LOQ}

The system precision of this GC-HS method is expressed in the term of \% RSD of the data. System precision at LOQ concentration has been demonstrated by injecting the six replicates of standard solutions. The obtained \%RSD was not more than $15 \%$. All values and chromatograms are shown in Table 7.

\section{Robustness}

To determine the robustness of this GC-HS method, the \%RSD was checked, to change any two method parameters from the initial conditions. Those parameters are column oven temperature $35^{\circ} \mathrm{C}$ and $45{ }^{\circ} \mathrm{C}$, 


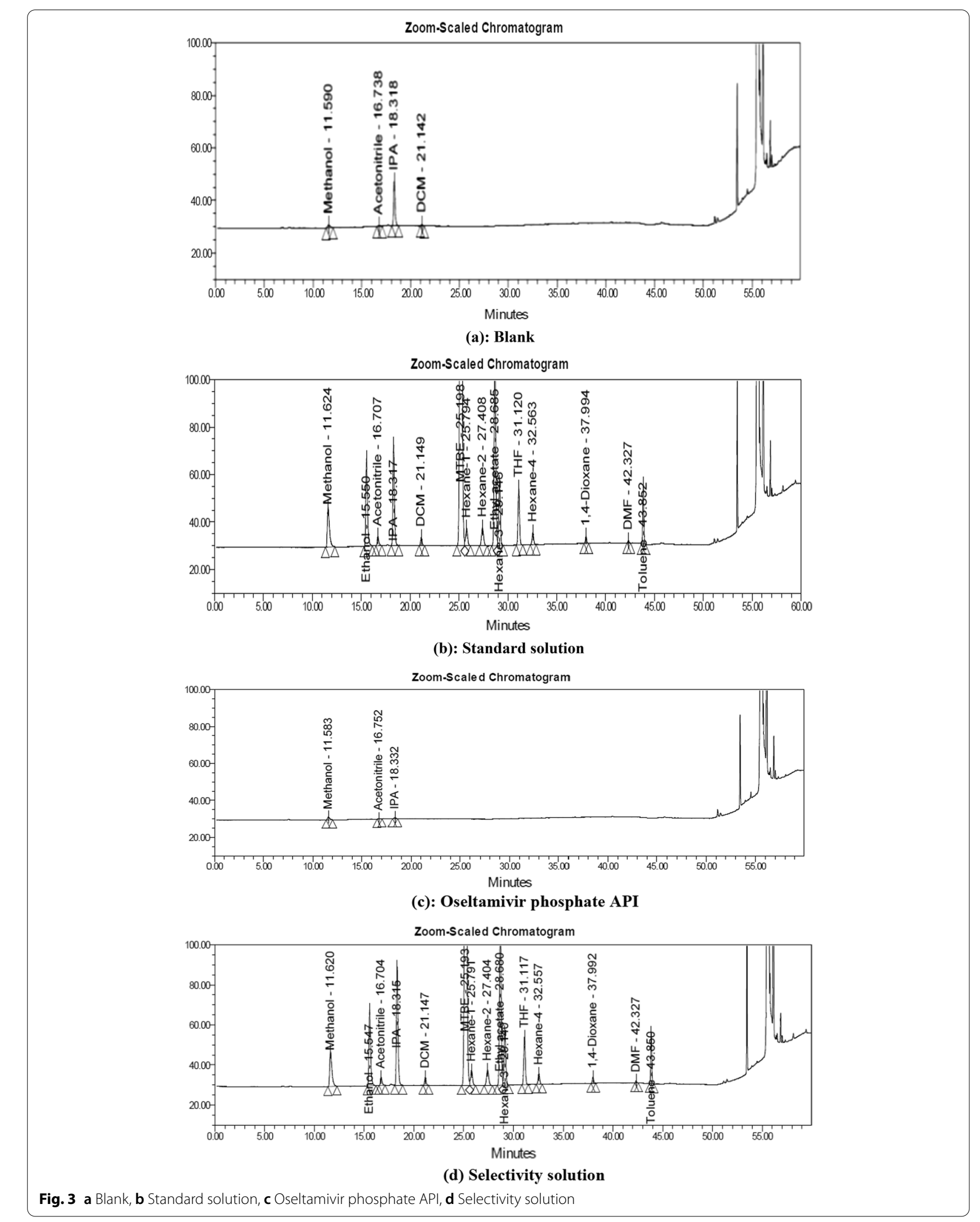


Table 1 Specificity and Selectivity data

\begin{tabular}{lllll}
\hline S. No & Name & $\begin{array}{l}\text { Retention times of each } \\
\text { solvent }\end{array}$ & $\begin{array}{l}\text { Retention times of solvents in } \\
\text { selectivity solution }\end{array}$ & $\begin{array}{l}\text { Resolution between solvent } \\
\text { peaks in selectivity solution }\end{array}$ \\
\hline 1 & Methanol & 11.61 & 11.62 & - \\
2 & Ethanol & 15.55 & 15.55 & 10.9 \\
3 & Acetonitrile & 16.7 & 16.7 & 3.8 \\
4 & IPA & 18.31 & 18.32 & 5.5 \\
5 & DCM & 21.15 & 21.15 & 10.1 \\
6 & MTBE & 25.2 & 25.19 & 12.4 \\
7 & Hexane-1 & 25.79 & 25.79 & 1.6 \\
8 & Hexane-2 & 27.41 & 27.4 & 4.1 \\
9 & Ethyl acetate & 28.68 & 28.68 & 3.6 \\
10 & Hexane-3 & 29.14 & 29.14 & 1.4 \\
11 & THF & 31.12 & 31.12 & 6.3 \\
12 & Hexane-4 & 32.56 & 32.56 & 4.7 \\
13 & 1,4-Dioxane & 37.99 & 37.99 & 19.7 \\
14 & DMF & 42.33 & 42.33 & 19.3 \\
15 & Toluene & 43.85 & 43.85 & 7.3 \\
\hline
\end{tabular}

Table 2 System precision data

\begin{tabular}{|c|c|c|c|c|c|c|c|}
\hline \multirow[t]{2}{*}{ No of injections } & \multicolumn{7}{|c|}{ Peak areas } \\
\hline & Methanol & Ethanol & Acetonitrile & IPA & DCM & MTBE & Ethyl acetate \\
\hline 1 & 286.66 & 454.05 & 46.85 & 471.52 & 36.17 & 4470.86 & 982.84 \\
\hline 2 & 258.7 & 443.7 & 45.24 & 462.03 & 35.3 & 4406.22 & 964.7 \\
\hline 3 & 260.6 & 451.7 & 46.03 & 469.01 & 35.7 & 4407.46 & 970.3 \\
\hline 4 & 267.99 & 454.64 & 45.92 & 473.38 & 35.78 & 4498.06 & 976.72 \\
\hline 5 & 282.98 & 463.53 & 47.53 & 482.04 & 36.54 & 4473.67 & 992.4 \\
\hline 6 & 286.41 & 464.41 & 48.15 & 487.92 & 36.26 & 4421.39 & 984.88 \\
\hline Avg & 273.9 & 455.3 & 46.6 & 474.3 & 36 & 4446.3 & 978.6 \\
\hline STDEV & 12.997 & 7.749 & 1.092 & 9.301 & 0.449 & 39.413 & 10.134 \\
\hline$\%$ RSD & 4.7 & 1.7 & 2.3 & 2 & 1.2 & 0.9 & 1 \\
\hline \multirow[t]{2}{*}{ No of injections } & \multicolumn{7}{|c|}{ Peak areas } \\
\hline & \multicolumn{2}{|c|}{ THF } & 1,4-Dioxane & \multicolumn{2}{|l|}{ DMF } & Toluene & Hexanes \\
\hline 1 & \multicolumn{2}{|c|}{266.16} & 27.79 & \multicolumn{2}{|l|}{8.37} & 192.18 & 613.03 \\
\hline 2 & \multicolumn{2}{|c|}{262.23} & 27.2 & \multicolumn{2}{|l|}{7.67} & 187.52 & 607.53 \\
\hline 3 & \multicolumn{2}{|c|}{263.57} & 27.42 & \multicolumn{2}{|l|}{7.44} & 188.88 & 606.39 \\
\hline 4 & \multicolumn{2}{|c|}{265.19} & 27.62 & \multicolumn{2}{|l|}{8.03} & 191.38 & 627.26 \\
\hline 5 & \multicolumn{2}{|c|}{268.5} & 28.33 & \multicolumn{2}{|l|}{7.99} & 194.33 & 608.18 \\
\hline 6 & \multicolumn{2}{|c|}{265.31} & 28.98 & \multicolumn{2}{|l|}{9.87} & 199.53 & 604.22 \\
\hline Avg & \multicolumn{2}{|c|}{265.2} & 27.9 & \multicolumn{2}{|l|}{8.2} & 192.3 & 611.1 \\
\hline STDEV & \multicolumn{2}{|c|}{2.158} & 0.658 & \multicolumn{2}{|l|}{0.865} & 4.285 & 8.434 \\
\hline$\%$ RSD & \multicolumn{2}{|c|}{0.8} & 2.4 & \multicolumn{2}{|l|}{10.5} & 2.2 & 1.4 \\
\hline
\end{tabular}

Bold values indicate better results than other filtering methods 
Table 3 Method precision data

\begin{tabular}{|c|c|c|c|c|c|c|c|}
\hline \multirow[t]{2}{*}{ No of injections } & \multicolumn{7}{|c|}{ Peak areas } \\
\hline & Methanol & Ethanol & Acetonitrile & IPA & DCM & MTBE & Ethyl acetate \\
\hline 1 & 3069.05 & 5104.35 & 430.81 & 7082.74 & 710.01 & 4878.18 & 5011.39 \\
\hline 2 & 3137.88 & 5313.08 & 439.01 & 7281.68 & 720.92 & 4954.33 & 5122.94 \\
\hline 3 & 2759.24 & 4859.6 & 408.87 & 6716.3 & 686.26 & 4839.57 & 4888.58 \\
\hline 4 & 2796.26 & 4917.64 & 412.47 & 6724.73 & 685.49 & 4884.04 & 4897.69 \\
\hline 5 & 2785.96 & 4913.64 & 414.77 & 6725.74 & 694.45 & 4910.43 & 4912.97 \\
\hline 6 & 2764.73 & 4867.66 & 410.66 & 6519.87 & 674.61 & 4893.74 & 4886.57 \\
\hline Avg & 2885.5 & 4996 & 419.4 & 6841.8 & 695.3 & 4893.4 & 4953.4 \\
\hline STDEV & 170.754 & 179.041 & 12.421 & 282.17 & 17.202 & 37.999 & 95.407 \\
\hline$\%$ RSD & 5.9 & 3.60 & 3.00 & 4.10 & 2.5 & 0.80 & 1.90 \\
\hline \multirow[t]{2}{*}{ No of injections } & \multicolumn{7}{|c|}{ Peak areas } \\
\hline & \multicolumn{2}{|c|}{ THF } & 1,4-Dioxane & \multicolumn{2}{|l|}{ DMF } & Toluene & Hexanes \\
\hline 1 & \multicolumn{2}{|c|}{712.97} & 391.13 & \multicolumn{2}{|l|}{965.73} & 913.78 & 283.1 \\
\hline 2 & \multicolumn{2}{|c|}{727.4} & 401.8 & \multicolumn{2}{|l|}{1037.48} & 949.3 & 287.78 \\
\hline 3 & \multicolumn{2}{|c|}{700.78} & 370.48 & \multicolumn{2}{|l|}{803.85} & 863.99 & 280.08 \\
\hline 4 & \multicolumn{2}{|c|}{702.69} & 374.41 & \multicolumn{2}{|l|}{836.6} & 868.76 & 287.89 \\
\hline 5 & \multicolumn{2}{|c|}{705.31} & 372.42 & \multicolumn{2}{|l|}{812.12} & 868.77 & 289.88 \\
\hline 6 & \multicolumn{2}{|c|}{702.05} & 369.11 & \multicolumn{2}{|l|}{776.18} & 859.91 & 287.38 \\
\hline Avg & \multicolumn{2}{|c|}{708.5} & 379.9 & \multicolumn{2}{|l|}{872} & 887.4 & 286 \\
\hline STDEV & \multicolumn{2}{|c|}{10.221} & 13.394 & \multicolumn{2}{|l|}{104.722} & 36.127 & 3.665 \\
\hline$\%$ RSD & \multicolumn{2}{|c|}{1.4} & 3.5 & \multicolumn{2}{|l|}{12.0} & 4.1 & 1.3 \\
\hline
\end{tabular}

Bold values indicate better results than other filtering methods

Table $4 \mathrm{LOD}$ and LOQ data

\begin{tabular}{llllll}
\hline Name of OVI & \multicolumn{2}{l}{ LOD } & & \multicolumn{2}{l}{ LOQ } \\
\cline { 2 - 3 } \cline { 6 - 6 } \cline { 5 - 6 } & Conc. $(\mathbf{p p m})$ & S/N ratio & & Conc. (ppm) & S/N ratio \\
\hline Methanol & 16.52 & 10.5 & & 54.06 & 10.5 \\
Ethanol & 14.53 & 4.5 & & 48.6 & 4.5 \\
Acetonitrile & 20.78 & 27.1 & & 66.49 & 27.1 \\
IPA & 6.01 & 4.3 & & 20.03 & 4.3 \\
DCM & 18.04 & 3.3 & & 54.13 & 3.3 \\
MTBE & 1.5 & 3.2 & & 5.51 & 3.2 \\
Ethyl acetate & 6 & 3.3 & & 20.01 & 3.3 \\
THF & 3.27 & 3.1 & & 10.89 & 3.1 \\
1,4-Dioxane & 15.2 & 3.5 & & 49.4 & 3.5 \\
DMF & 80.13 & 3.4 & & 267.09 & 3.4 \\
Toluene & 4.05 & 4.7 & & 13.49 & 4.7 \\
Hexanes & 0.88 & 4.4 & & 2.94 & 4.4 \\
\hline
\end{tabular}

and injector temperature is $175{ }^{\circ} \mathrm{C}$ and $185{ }^{\circ} \mathrm{C}$. The results are presented in Table 8 . Finally, in two changed method parameters, the \%RSD was not more than $15.0 \%$ for each organic volatile impurity.
Table 5 Linearity data

\begin{tabular}{ll}
\hline Name of OVI & Correlation(r) \\
\hline Methanol & 0.9996 \\
Ethanol & 0.9998 \\
Acetonitrile & 0.9993 \\
IPA & 0.9998 \\
DCM & 0.9994 \\
MTBE & 0.9999 \\
Ethyl acetate & 0.9998 \\
THF & 0.9998 \\
1,4-Dioxane & 0.9997 \\
DMF & 0.997 \\
Toluene & 0.9997 \\
Hexanes & 0.9999 \\
\hline
\end{tabular}

\section{Ruggedness}

Ruggedness is the ability of the chemical measurement process to resist changes in the test results when subjected to minor changes in environmental and method procedural variables, laboratories, personnel, 

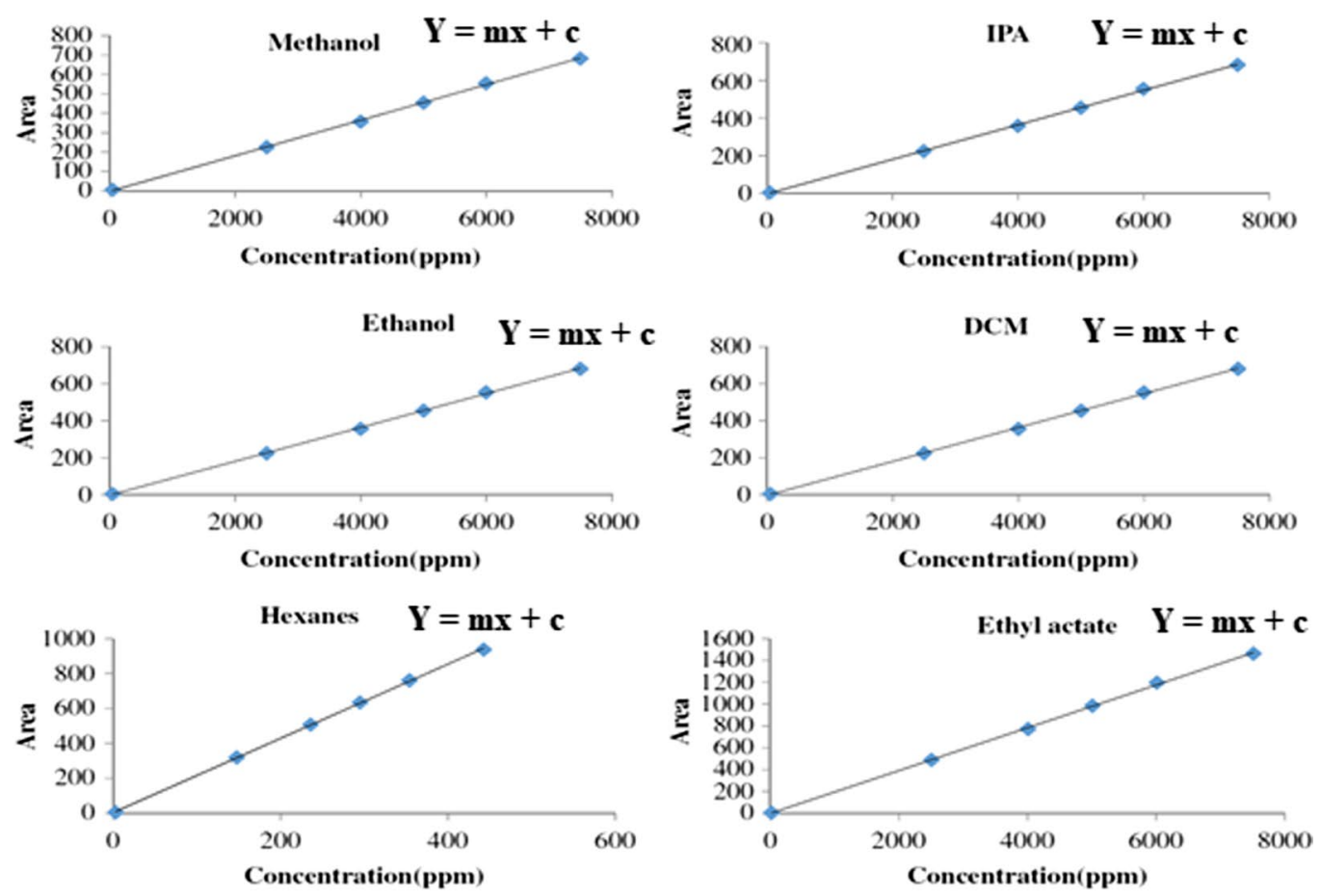

(a): Correlation graph
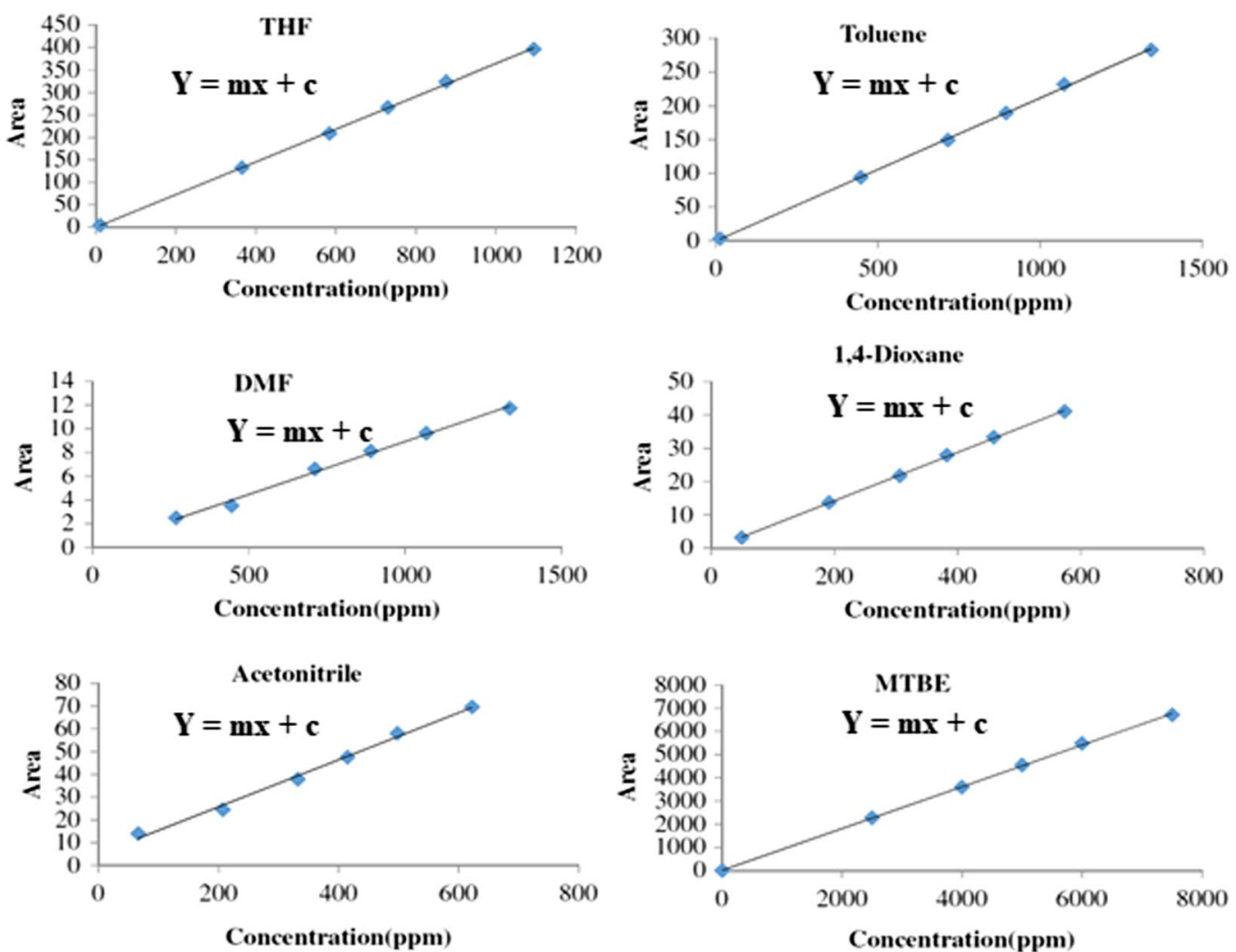

(b): Correlation graph

Fig. 4 a Correlation graph, b Correlation graph 
Table 6 Accuracy data

\begin{tabular}{lclll}
\hline Name of OVI & $\begin{array}{l}\text { LOQ } \\
\text { recovery } \\
(\mathbf{\% )}\end{array}$ & $\begin{array}{l}\mathbf{5 0 \%} \\
\text { Recovery }\end{array}$ & $\begin{array}{l}\mathbf{1 0 0 \%} \\
\text { Recovery }\end{array}$ & $\begin{array}{l}\mathbf{1 5 0 \%} \\
\text { Recovery }\end{array}$ \\
\hline Methanol & 109.6 & 103.4 & 100.1 & 100.6 \\
Ethanol & 102.5 & 105.3 & 102.6 & 102.5 \\
Acetonitrile & 88.3 & 113.5 & 99.1 & 101.7 \\
IPA & 85.3 & 103.2 & 102.1 & 101.5 \\
DCM & 110.2 & 104.7 & 101.5 & 101.1 \\
MTBE & 89.5 & 105 & 101.7 & 99.5 \\
Ethyl acetate & 100.5 & 105.2 & 101.7 & 100.9 \\
THF & 94.4 & 103.7 & 100.3 & 99.3 \\
1,4-Dioxane & 110.7 & 105.5 & 101.3 & 100.7 \\
DMF & 108.8 & 100.4 & 96.1 & 97 \\
Toluene & 98.7 & 105.8 & 101.8 & 101.3 \\
Hexanes & 73.7 & 104.9 & 102.1 & 98.5 \\
\hline
\end{tabular}

etc. Ruggedness has been established by separate six analyses of a single batch of the sample prepared by two different analysts on different days. Overall RSD of residual solvents was found out to be less than $15 \%$ as per AOAC guidelines and USP general chapter $<1467>$. According to this data, our method was rugged. All values are presented in Table 9.

\section{Solution stability}

Stability of six organic volatile impurities standard and Oseltamivir phosphate API sample prepared in NMP as a diluent. Three-time intervals (After 12 Hours $(\mathrm{H})$, after $24 \mathrm{H}$ and after $48 \mathrm{H}$ ) have been observed from the Initial time. The standard solutions were prepared on the same day and keep them at room temperature. Injected standard organic volatile solution at Initial, after $12 \mathrm{H}$, after $12 \mathrm{H}$ and after $48 \mathrm{H}$. Then the calculated \% of solution stability at each time point, compared with Initial time area of each organic volatile impurity. The $\%$ of solution stability is $100 \pm 15 \%$ as per in-house established solution stability data. From these solution stability results; twelve organic volatile impurities standards were stable up to $48 \mathrm{~h}$. The corresponding data are presented in Table 10.

\section{Application of the proposed method (analysis of Oseltamivir phosphate tablet)}

The proposed method was evaluated by the assay of commercially available Oseltamivir phosphate tablet (Oseltamivir $75 \mathrm{mg}$ ) for the quantitative deamination of organic twelve volatile impurities present in it. The obtained results should have within our proposed specifications. This revealed that the concentration of these twelve organic volatile impurities has presented

Table 7 System precision data at $L O Q$

\begin{tabular}{|c|c|c|c|c|c|c|c|c|}
\hline \multirow[t]{2}{*}{ No of injections } & \multicolumn{8}{|c|}{ Peak areas } \\
\hline & \multicolumn{2}{|c|}{ Methanol } & Ethanol & Acetonitrile & IPA & DCM & MTBE & Ethyl acetate \\
\hline 1 & \multicolumn{2}{|l|}{9.9} & 4.35 & 13.44 & 4.64 & 3.43 & 4.73 & 4.76 \\
\hline 2 & \multicolumn{2}{|l|}{11.13} & 3.53 & 14.29 & 5.74 & 4.06 & 4.62 & 4.54 \\
\hline 3 & \multicolumn{2}{|l|}{10.78} & 3.49 & 13.71 & 5.51 & 3.76 & 4.58 & 4.94 \\
\hline 4 & \multicolumn{2}{|l|}{10.29} & 3.93 & 13.7 & 5.11 & 3.21 & 4.46 & 4.24 \\
\hline 5 & \multicolumn{2}{|l|}{10.03} & 3.37 & 13.32 & 6.13 & 3.15 & 5.01 & 4.47 \\
\hline 6 & \multicolumn{2}{|l|}{10.84} & 3.55 & 15.14 & 5.06 & 3.49 & 4.79 & 4.27 \\
\hline Avg & \multicolumn{2}{|l|}{10.5} & 3.7 & 13.9 & 5.4 & 3.5 & 4.7 & 4.5 \\
\hline STDEV & \multicolumn{2}{|l|}{0.493} & 0.369 & 0.679 & 0.535 & 0.344 & 0.192 & 0.274 \\
\hline$\%$ RSD & 4.7 & & 10 & 4.9 & 9.9 & 9.8 & 4.1 & 6.1 \\
\hline \multirow[t]{2}{*}{ No of injections } & & \multicolumn{7}{|c|}{ Peak areas } \\
\hline & \multicolumn{3}{|c|}{ THF } & 1,4-Dioxane & DMF & \multicolumn{2}{|r|}{ Toluene } & Hexanes \\
\hline 1 & \multicolumn{2}{|r|}{3.92} & \multicolumn{2}{|r|}{3.22} & 2.5 & \multicolumn{2}{|r|}{2.81} & 5.08 \\
\hline 2 & \multicolumn{2}{|r|}{3.75} & \multicolumn{2}{|r|}{2.89} & 2.42 & \multicolumn{2}{|r|}{2.77} & 5.87 \\
\hline 3 & \multicolumn{2}{|r|}{3.71} & \multicolumn{2}{|r|}{2.85} & 2.55 & \multicolumn{2}{|r|}{2.53} & 5.84 \\
\hline 4 & \multicolumn{2}{|r|}{3.67} & \multicolumn{2}{|r|}{2.9} & 1.87 & \multicolumn{2}{|r|}{2.75} & 4.78 \\
\hline 5 & \multicolumn{2}{|r|}{3.59} & \multicolumn{2}{|r|}{2.67} & 2.25 & \multicolumn{2}{|r|}{2.47} & 4.91 \\
\hline 6 & \multicolumn{2}{|r|}{3.46} & \multicolumn{2}{|r|}{2.97} & 2.31 & \multicolumn{2}{|r|}{2.42} & 4.82 \\
\hline Avg & & 3.7 & & 2.9 & 2.3 & & 2.6 & 5.2 \\
\hline STDEV & & 0.155 & & 0.18 & 0.246 & & 0.171 & 0.505 \\
\hline \%RSD & & 4.2 & & 6.2 & 10.7 & & 6.6 & 9.7 \\
\hline
\end{tabular}

Bold values indicate better results than other filtering methods 
Table 8 Robustness data

\begin{tabular}{|c|c|c|c|c|}
\hline \multirow{2}{*}{$\begin{array}{l}\text { Name of } \\
\text { organic } \\
\text { volatile } \\
\text { impurity }\end{array}$} & \multicolumn{4}{|c|}{$\%$ RSD for different method parameters } \\
\hline & $\begin{array}{l}\text { Column oven } \\
\text { Temp. } 35^{\circ} \mathrm{C}\end{array}$ & $\begin{array}{l}\text { Column oven } \\
\text { Temp. } 45^{\circ} \mathrm{C}\end{array}$ & $\begin{array}{l}\text { Injector } \\
\text { temp. } \\
175^{\circ} \mathrm{C}\end{array}$ & $\begin{array}{l}\text { Injector } \\
\text { temp. } \\
185^{\circ} \mathrm{C}\end{array}$ \\
\hline Methanol & 2.0 & 2.2 & 1.3 & 1.1 \\
\hline Ethanol & 2.6 & 1.8 & 1.3 & 0.4 \\
\hline Acetonitrile & 2.1 & 1.9 & 1.1 & 0.6 \\
\hline IPA & 2.6 & 2.1 & 1 & 0.5 \\
\hline DCM & 2.0 & 1.4 & 1 & 0.9 \\
\hline MTBE & 1.0 & 0.7 & 1.7 & 1 \\
\hline Ethyl acetate & 1.6 & 1.1 & 0.7 & 0.6 \\
\hline THF & 1.4 & 0.8 & 0.8 & 0.6 \\
\hline 1,4-Dioxane & 3.9 & 2.7 & 1.9 & 1.1 \\
\hline DMF & 13.5 & 10.3 & 5 & 5.2 \\
\hline Toluene & 3.9 & 2.5 & 1.2 & 0.7 \\
\hline Hexanes & 0.9 & 0.7 & 3.4 & 1.4 \\
\hline
\end{tabular}

Table 9 Ruggedness data

\begin{tabular}{llll}
\hline Name of OVI & \multicolumn{3}{l}{ \%RSD for different analysts and days } \\
\cline { 2 - 4 } & $\begin{array}{l}\text { Day-1 } \\
\text { Analyst-1 \&2 } \\
(\boldsymbol{n}=\mathbf{1 2})\end{array}$ & $\begin{array}{l}\text { Day-2 } \\
\text { Analyst-1 \&2 } \\
(\boldsymbol{n}=\mathbf{1 2})\end{array}$ & $\begin{array}{l}\text { Analyst-1 } \\
\text { Day-1 \&2 } \\
(\boldsymbol{n}=\mathbf{1 2})\end{array}$ \\
\hline Methanol & 1.5 & 2.6 & 6.2 \\
Ethanol & 2 & 0.6 & 2.7 \\
Acetonitrile & 12.62 & 12.1 & 13.9 \\
IPA & 2.2 & 0.8 & 3 \\
DCM & 2 & 0.5 & 2.1 \\
MTBE & 1.5 & 0.6 & 3.7 \\
Ethyl acetate & 1.5 & 0.2 & 1.8 \\
THF & 1.4 & 0.2 & 2.7 \\
1,4-Dioxane & 2.8 & 0.7 & 2.6 \\
DMF & 10.7 & 4.7 & 10 \\
Toluene & 2.4 & 0.4 & 3 \\
Hexanes & 1.5 & 1.4 & 5.3 \\
\hline
\end{tabular}

Oseltamivir phosphate tablet in ppm levels. The corresponding data are presented in Table 11.

\section{Discussion}

The development of an analytical method for the determination of twelve organic volatile impurities (Methanol, Ethanol, Acetonitrile, IPA, DCM, MTBE, Hexane fractions, EA, THF, 1,4-Dioxane, DMF, and Toluene) in Oseltamivir phosphate API and pharmaceutical dosage forms by GC-HS with flame ionization detector has received considerable attention in recent years because of their importance in quality control of API drug and its impurities. The goal of this study was to develop a simple rapid accurate and precise GC-HS method for the simultaneous determination of twelve organic volatile impurities using the most commonly employed DB-1, $60 \mathrm{~m}, 0.32 \mathrm{~mm}$, and $5.0 \mu \mathrm{m}$ column with flame ionization detector.

We got well resolution between twelve OVI's. The numbers of theoretical plates obtained for six organic volatile impurities were more than 2000, respectively, which indicates the efficiency of the column. The \% RSD was found not more than $15 \%$ for the system precision, Method precision, LOQ precision, Robustness, and Ruggedness. Linearity was observed over the concentration range of LOQ to $150 \%$ for twelve OVI's with a correlation coefficient $(\mathrm{r} 2=0.99)$. From these precision and Linearity data, our proposed method is precise and linear. We have obtained LOD and LOQ results for the twelve solvents very low level with acceptable USP s/n ratio. From LOD and LOQ result indicates the sensitivity of the method. The recovery was found to be $100 \pm 15 \%$ at $50 \%, 100 \%$, $150 \%$, and LOQ. We have reported the prepared standard, and sample solution in NMP is stable up to $48 \mathrm{~h}$. Our method is also applied to pharmaceutical dosage forms. The above all validated data indicates that the proposed GC-HS method is highly sensitive and accurate.

Table 10 Solution stability

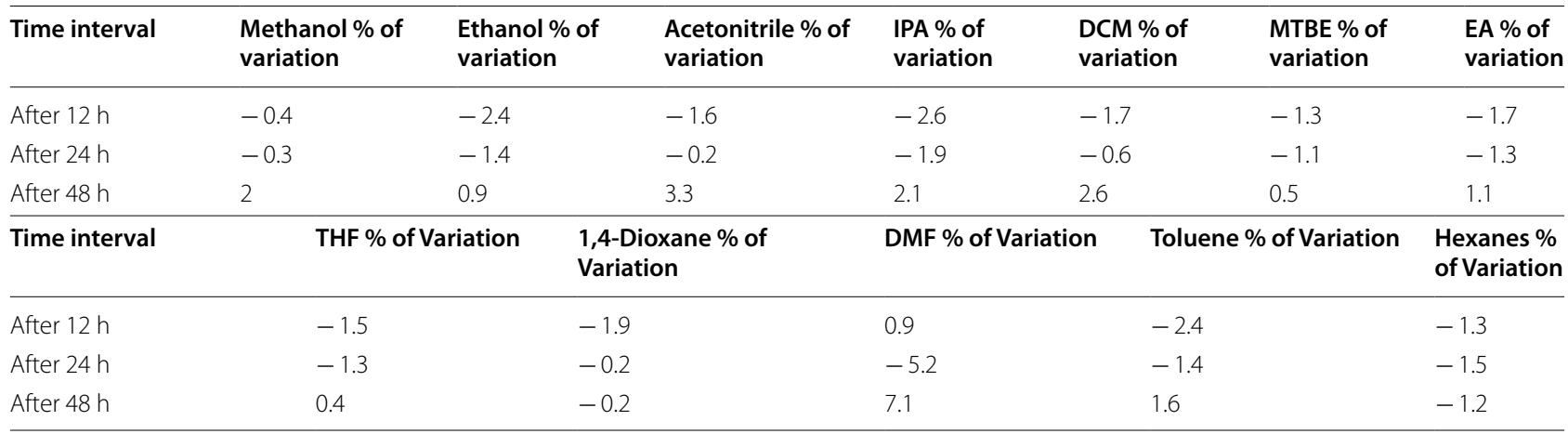


Table 11 Data for Oseltamivir phosphate tablet analysis

\begin{tabular}{llll}
\hline Si. No & Name of OVI & Results (ppm) & Approved values (ppm) \\
\hline 1 & Methanol & 108 & Not more than 3000 \\
2 & Ethanol & Not detected & Not more than 5000 \\
3 & Acetonitrile & 112 & Not more than 410 \\
4 & IPA & 120 & Not more than 5000 \\
5 & DCM & Not detected & Not more than 600 \\
6 & MTBE & Not detected & Not more than 5000 \\
7 & Ethyl acetate & Not detected & Not more than 5000 \\
8 & THF & Not detected & Not more than 720 \\
9 & 1,4-Dioxane & Not detected & Not more than 380 \\
10 & DMF & Not detected & Not more than 880 \\
11 & Toluene & Not detected & Not more than 890 \\
12 & Hexanes & Not detected & Not more than 290 \\
\hline
\end{tabular}

\section{Conclusions}

Reliable and effective gas chromatography coupled with flame ionization mode of detection dependent methodology to detect and quantify the residual chemical solvents Methanol, Ethanol, Acetonitrile, Isopropyl alcohol, Dichloromethane, MTBE, Hexanes, Ethyl acetate, THF, 1,4-Dioxane, DMF, Toluene in oseltamivir phosphate drug substance as well as in drug product. The validation parameters (linear, system suitability, quantification limit, detection limit, robustness, accuracy, precision, selectivity, ruggedness) for opted twelve residual chemical solvents were in line with $\mathrm{ICH}$ requirement and AOAC guidelines. Present results revealed that the quality of the oseltamivir phosphate sample can be evaluated using the methodology of gas chromatography proposed in this work. The method was found to be applicable for the routine analysis of the Oseltamivir phosphate API, and its pharmaceutical dosage forms in the pharmaceutical industry.

\begin{abstract}
Abbreviations
MTBE: Methyl tert-butyl ether; IPA: Isopropyl alcohol; THF: Tetra hydro furan; DMF: Dimethylformamide; DCM: Dichloromethane; EA: Ethyl acetate; API: Active pharmaceutical ingredient; $\mathrm{N}_{2}$ : Nitrogen; $\mathrm{ICH}$ : International Council for Harmonization; LOQ: Limit of quantification; LOD: Limit of detection; Ppm: Parts per million; AOAC: Association of Official Agricultural Chemists; RPHPLC: Reversed-phase high-performance liquid chromatography; LC: Liquid chromatography; GC-HS: Gas chromatography-head space; USA: United States of America; FID: Flame ionization detector; ${ }^{\circ} \mathrm{C}$ : Centigrade; NMP: N-methyl2-pyrrolidone; Psi: Pounds per square inch; mL: Milley letter; mg: Milly gram; RSD: Relative standard deviation; USP: United states pharmacopeia; s/n: Signal to noise; $\geq$ : Greater than or equal; OVI: Organic volatile impurities; H: Hours.
\end{abstract}

\section{Acknowledgements}

The authors thank Dr. P Shyamala and Dr. R Murali Krishna (Physical Chemistry Department, Andhra University, Visakhapatnam, Andhra Pradesh, India) and Dr.KMV Narayana Rao (GVK Biosciences Pvt. Ltd, Hyderabad, Telangana, India) for their encouragement, valuable inputs, and cooperation while carrying out this research work. The authors are also grateful to GVK Biosciences Pvt. Ltd,
Hyderabad, Telangana, India, for providing facilities to carry out this research work.

\section{Authors' contributions}

All authors read and approved the final manuscript.

Funding

Self-sufficient.

Availability of data and materials

From the literature and GVK Biosciences.

\section{Declarations}

\section{Ethics approval and consent to participate}

Our proposed work is not related to Bio work. This is completely Impurity analysis by a new analytical technique (GC-HS). In the IRB guidelines like 21 CFR 50.24 and 45 CFR, Part 46" is mentioned Human, Bio, Clinical trials work. According to the above guideline, our work is not related to Human, Bio, Clinical trials work. This is completely new analytical method development work. So, I hope do need the "Ethics approval and consent to participate".

\section{Consent for publication}

Our proposed work is not related to Bio and Clinical trials work. So, "Consent for publication" is not applicable.

\section{Competing interests}

Not applicable.

\section{Author details}

${ }^{1}$ GVK Biosciences Pvt. Ltd, Hyderabad, Telangana, India. ${ }^{2}$ Department of Physical Chemistry, Andhra University, Visakhapatnam, India. ${ }^{3}$ Department of Physical and Nuclear Chemistry and Department of Chemical Oceanography, Andhra University, Visakhapatnam, Andhra Pradesh, India. ${ }^{4}$ Krishna, India.

Received: 20 August 2021 Accepted: 4 January 2022

Published online: 28 January 2022

\section{References}

1. Bonadio F, Margot P, Delémont O, Esseiva P (2009) Optimization of HSSPME/GC-MS analysis and its use in the profiling of illicit ecstasy tablets (Part 1). Forensic Sci Int 187:73

2. Li J, Shao S, Solorzano M, Allmaier GJ, Kurtulik PT (2009) Determination of the residual ethanol in hydroalcoholic sealed hard gelatin capsules by static headspace gas chromatography with immiscible binary solvents. J Chromatogr A 1216:3328

3. Barro R, Regueiro J, Llompart M, Garcia-Jares C (2009) Analysis of industrial contaminants in indoor air: part 1. Volatile organic compounds, carbonyl compounds, polycyclic aromatic hydrocarbons and polychlorinated biphenyls. J Chromatogr A 1216:540

4. Dmorcillo Y, Cai Y, Bayona JM (1995) Rapid determination of methyltin compounds in aqueous samples using solid phase microextraction and capillary gas chromatography following in-situ derivatization with sodium tetraethylborate. J High Resol Chromatogr 18:776

5. ICH Harmonized Tripartite Guidelines for Residual Solvents (1997) Step 4.

6. Fliszar K, Markwiggins J, Pignoli CM (2004) Analysis of organic volatile impurities in pharmaceutical excipients by static headspace capillary gas chromatography. J Chromatogr 1027:83

7. $\mathrm{ICH}$ harmonized guideline (2016) impurities: a guideline for residual Solvents Q3C(R6), International Council for harmonization of technical requirements for pharmaceuticals for human use.

8. Malipatil SM, Jahan K, Patil SK (2011) Development and validation of RP-HPLC method for the determination of oseltamivir phosphate in bulk drug and dosage. Indo Glob J Pharm Sci 1:57-62

9. Raghuram P, Soma Raju IV, Reddy R, Sriramulu J (2008) Stability indicating LC method for oseltamivir phosphate. Anal Chem 7:617-624 
10. Chatpalliwar VA, Upmanyu N, Porwal PK (2012) Validated gradient stability-indicating HPLC method for determining diltiazem hydrochloride and related substances in bulk drug and novel tablet formulation. J Pharm Anal 2:226-237

11. ICH. Guidelines (1996) Q2B Validation of Analytical Procedures Methodology in Proceedings of ICH. Rockville, USA p. 1-10.

\section{Publisher's Note}

Springer Nature remains neutral with regard to jurisdictional claims in published maps and institutional affiliations.

\section{Submit your manuscript to a SpringerOpen ${ }^{\circ}$ journal and benefit from:}

- Convenient online submission

- Rigorous peer review

- Open access: articles freely available online

- High visibility within the field

- Retaining the copyright to your article

Submit your next manuscript at $\boldsymbol{\Delta}$ springeropen.com 